\title{
Serotonergic Modulation of Locomotion in Zebrafish-Endogenous Release and Synaptic Mechanisms
}

\author{
Jens Peter Gabriel, ${ }^{\star}$ Riyadh Mahmood, ${ }^{1 \star}$ Alexandros Kyriakatos, ${ }^{1}$ Iris Söll, ${ }^{2,3}$ Giselbert Hauptmann, ${ }^{2,3}$ \\ Ronald L. Calabrese, ${ }^{4}$ and Abdeljabbar El Manira ${ }^{1}$ \\ ${ }^{1}$ Department of Neuroscience, Karolinska Institutet, SE-171 77 Stockholm, Sweden, ${ }^{2}$ School of Life Sciences, Södertörns Högskola, SE-141 89 Huddinge, \\ Sweden, ${ }^{3}$ Department of Biosciences and Nutrition, Novum, Karolinska Institutet, SE-141 57 Huddinge, Sweden, and ${ }^{4}$ Department of Biology, Emory \\ University, Atlanta, Georgia 30322
}

Serotonin (5-HT) plays an important role in shaping the activity of the spinal networks underlying locomotion in many vertebrate preparations. At larval stages in zebrafish, 5-HT does not change the frequency of spontaneous swimming; and it only decreases the quiescent period between consecutive swimming episodes. However, it is not known whether 5-HT exerts similar actions on the locomotor network at later developmental stages. For this, the effect of 5-HT on the fictive locomotor pattern of juvenile and adult zebrafish was analyzed. Bath-application of 5-HT (1-20 $\mu \mathrm{M})$ reduced the frequency of the NMDA-induced locomotor rhythm. Blocking removal from the synaptic cleft with the reuptake inhibitor citalopram had similar effects, suggesting that endogenous serotonin is modulating the locomotor pattern. One target for this modulation was the mid-cycle inhibition during locomotion because the IPSPs recorded in spinal neurons during the hyperpolarized phase were increased both in amplitude and occurrence by 5-HT. Similar results were obtained for IPSCs recorded in spinal neurons clamped at the reversal potential of excitatory currents $(0 \mathrm{mV})$. 5-HT also slows down the rising phase of the excitatory drive recorded in spinal cord neurons when glycinergic inhibition is blocked. These results suggest that the decrease in the locomotor burst frequency induced by 5-HT is mediated by a potentiation of mid-cycle inhibition combined with a delayed onset of the subsequent depolarization.

\section{Introduction}

Modulatory inputs acting via G-protein coupled receptors provide neuronal circuits with the flexibility required to adapt their activity to internal and external changes. In the spinal cord, the network producing locomotor activity is subject both to intrinsic and extrinsic neuromodulation. In many vertebrates, 5-HT is considered to play an important role in organizing the locomotor pattern and can profoundly modulate the motor output. In some preparations the cellular and synaptic mechanisms underlying the 5-HT modulation of the network activity have begun to be elucidated. In Xenopus tadpoles, fictive locomotion can be induced by skin stimulation, and application of 5-HT increases the locomotor burst duration, depresses mid-cycle inhibition, and modulates NMDA receptors (Sillar et al., 1992; ScrymgeourWedderburn et al., 1997; Sillar et al., 1998). In the newborn mammalian spinal cord, 5-HT is important for generating a stable locomotor pattern induced pharmacologically (Cazalets et al., 1992; Kiehn and Kjaerulff, 1996; Liu and Jordan, 2005) (see also

\footnotetext{
Received April 27, 2009; revised July 2, 2009; accepted July 8, 2009.

This work was supported by the Swedish Research Council, Söderberg Foundation, Wings For Life Spinal Cord Research Foundation, the European Commission (Health-F2-2007-201144), Christopher and Dana Reeve Foundation, and the Karolinska Institutet. J.P.G. was supported by a fellowship from the German Science Foundation. We thank Dr. Russell Hill for comments on this manuscript.

*J.P.G. and R.M. contributed equally to this work.

Corresponding author: Abdel El Manira, Department of Neuroscience, Karolinska Institutet, SE-171 77 Stockholm, Sweden. E-mail: Abdel.ElManira@ki.se.

DOI:10.1523/JNEUROSCI.1978-09.2009

Copyright $\odot 2009$ Society for Neuroscience $\quad 0270-6474 / 09 / 2910387-09 \$ 15.00 / 0$
}

Liu et al., 2009). The change in the locomotor frequency seems to depend of the type of 5-HT receptor activated (Beato and Nistri, 1998; Bracci et al., 1998; Madriaga et al., 2004). In the adult spinal cord of the lamprey, 5-HT is endogenously released from a ventromedial plexus and decreases the locomotor frequency by changing the intrinsic properties of neurons and synaptic transmission (Wallén et al., 1989a,b; Zhang et al., 1996; El Manira et al., 1997; Schwartz et al., 2005).

The zebrafish has emerged as a new amenable model system in which the organization, function and modulation of the locomotor network can be studied early in development and in the adult system (Masino and Fetcho, 2005; McDearmid and Drapeau, 2006; Fetcho et al., 2008; Gabriel et al., 2008). In the spinal cord of both larval and adult zebrafish, 5-HT innervation arises from descending raphe projections and intraspinal neurons (Van Raamsdonk et al., 1996; Kaslin and Panula, 2001; McLean and Fetcho, 2004). At the larval stage, 5-HT does not affect the frequency of the spontaneous swimming and only decreases the quiescent period between consecutive swimming episodes (Brustein and Drapeau, 2005). This is in contrast to what has been reported in other model systems and raises the question whether the lack of effect on the locomotor frequency is only apparent at larval stages or if it persists in the adult.

In this study, we have used a recently developed in vitro preparation of the adult zebrafish brainstem/spinal cord to examine the 5-HT modulation of the frequency of fictive swimming and the underlying mechanisms. We show that 5-HT is endogenously released during fictive locomotion and decreases the frequency of 
the rhythm, in contrast to its effect in larvae. Another important finding of this study is that during locomotion, the onphase depolarization and mid-cycle inhibition are mediated by phasic and alternating excitatory and inhibitory currents, respectively. Finally, 5-HT seems to decrease the frequency of the locomotor frequency by increasing the mid-cycle inhibition and delaying the onset of the following on-phase excitation.

\section{Materials and Methods}

Zebrafish preparations. Zebrafish ( $\mathrm{ABC}$ and $\mathrm{AB} /$ Tuebingen strains) were raised and kept according to established procedures (Westerfield, 2000). All experimental protocols were approved by the Animal Research Ethical Committee, Stockholm.

The preparation was performed as described previously (Gabriel et al., 2008) with slight modifications. Zebrafish (age 6-20 weeks) were cold-anesthetized and dissected in a slush of frozen fish saline containing in mM: 134 $\mathrm{NaCl}, 2.9 \mathrm{KCl}, 2.1 \mathrm{CaCl}_{2}, 1.2 \mathrm{MgCl}_{2}, 10$ HEPES, and 10 glucose, with $\mathrm{pH}$ adjusted to 7.8 with $\mathrm{NaOH}$, and osmolarity of $290 \mathrm{mOsm}$. The fish were pinned down ventral side up, quickly eviscerated, and then glued dorsal side up to a Petri dish. The skull was opened and the brain was cut at the level of the tectum, sparing the caudal part of the midbrain. The dorsal and lateral musculature was removed up to the caudal end of the dorsal fin, leaving the musculature at the tail intact. Here, the skin was pulled away to expose the muscle fibers for electromyographic (EMG) recordings. With two pairs of forceps the vertebrae overlying the spinal cord were bent aside and pulled out. To facilitate electrode penetration, small gashes were made in the meninges overlying the spinal cord with a sharpened tungsten pin. The entire spinal cord including the hindbrain and the caudal musculature was then cut out together with the vertebral column and transferred to the recording chamber. The preparation was placed lateral side up for patch-clamp recordings (Fig. $1 A$ ) or ventral side up for extracellular recordings and fixed in place with Vaseline. During the experiments, the preparations were continuously perfused with oxygenated fish saline at room temperature. The following drugs were stored as stock solutions at $-20^{\circ} \mathrm{C}$ and were dissolved to their final concentrations in physiological solution before their application: NMDA $(30-50 \mu \mathrm{M})$, 5-HT $(1-20 \mu \mathrm{M})$, and strychnine $(0.5 \mu \mathrm{M})$, all from Tocris-Cookson, and TTX $(0.5 \mu \mathrm{M}$, Sigma-Aldrich).

Backfills of motoneurons. Some experiments were performed on identified dorsal secondary motoneurons. For this, small crystals of the fluorescent tracer tetramethylrhodamine dextran (3000 MW, Invitrogen) were dissolved in distilled water. Sharpened tungsten pins were moved through the viscous dye solution so that the dye solidified on the tip. Animals were anesthetized in tricaine methanesulfonate (MS-222 0.03\%, Sigma-Aldrich) and placed on a glass Petri
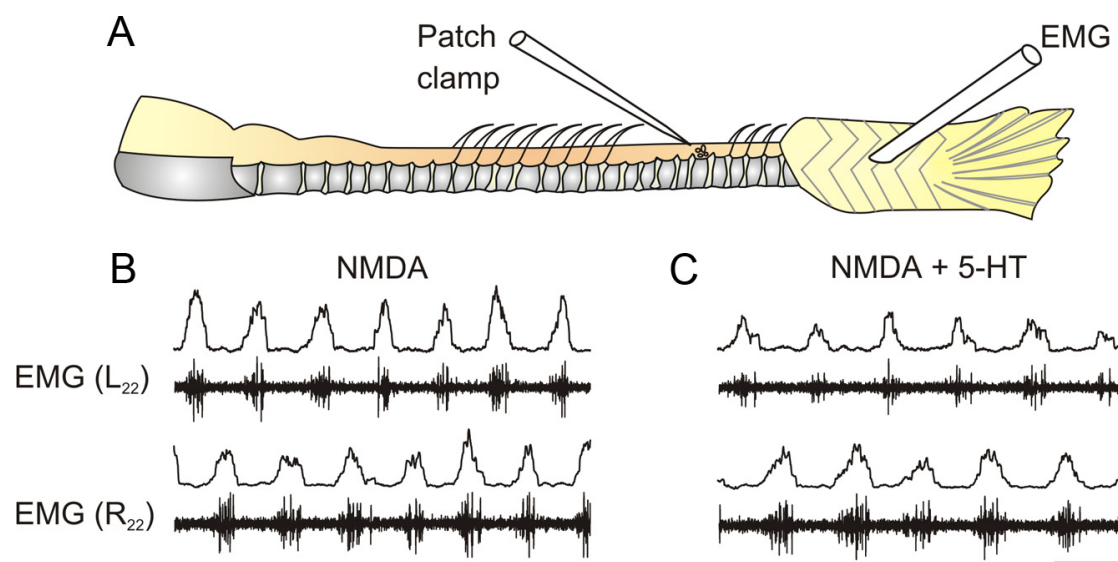

C NMDA + 5-HT
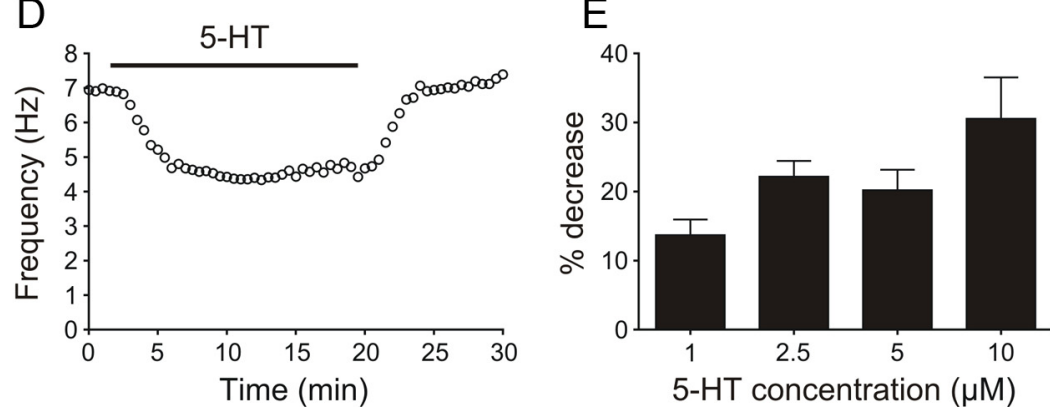

Figure 1. 5 -HT decreases the locomotor cycle frequency. $A$, Schematic drawing of the preparation. $B$, Locomotor pattern is generated in the in vitro hindbrain/spinal cord preparation by perfusion of NMDA $(40 \mu \mathrm{M})$. Throughout the figures, the raw EMG trace is shown together with the smoothed rectified trace. $\boldsymbol{C}$, When $5-\mathrm{HT}(5 \mu \mathrm{m})$ is added, the locomotor frequency decreases. $\boldsymbol{D}$, Time course of the decrease in the locomotor frequency (fictive locomotion induced by $40 \mu \mathrm{MNMDA}$ ) in $5-\mathrm{HT}(10 \mu \mathrm{M})$. $\boldsymbol{E}$, Decrease in the locomotor frequency at different 5 -HT concentrations. Addition of $1 \mu \mathrm{m} 5$-HT decreased the locomotor frequency induced by $30-50 \mu \mathrm{m} \mathrm{NMDA} \mathrm{by} 13.7 \%$ ( $n=$ 12). The decrease in $2.5,5$, and $10 \mu \mathrm{m} 5$-HT was $22.1 \%(n=11), 20.2 \%(n=14)$ and $30.5 \%(n=2)$, respectively.
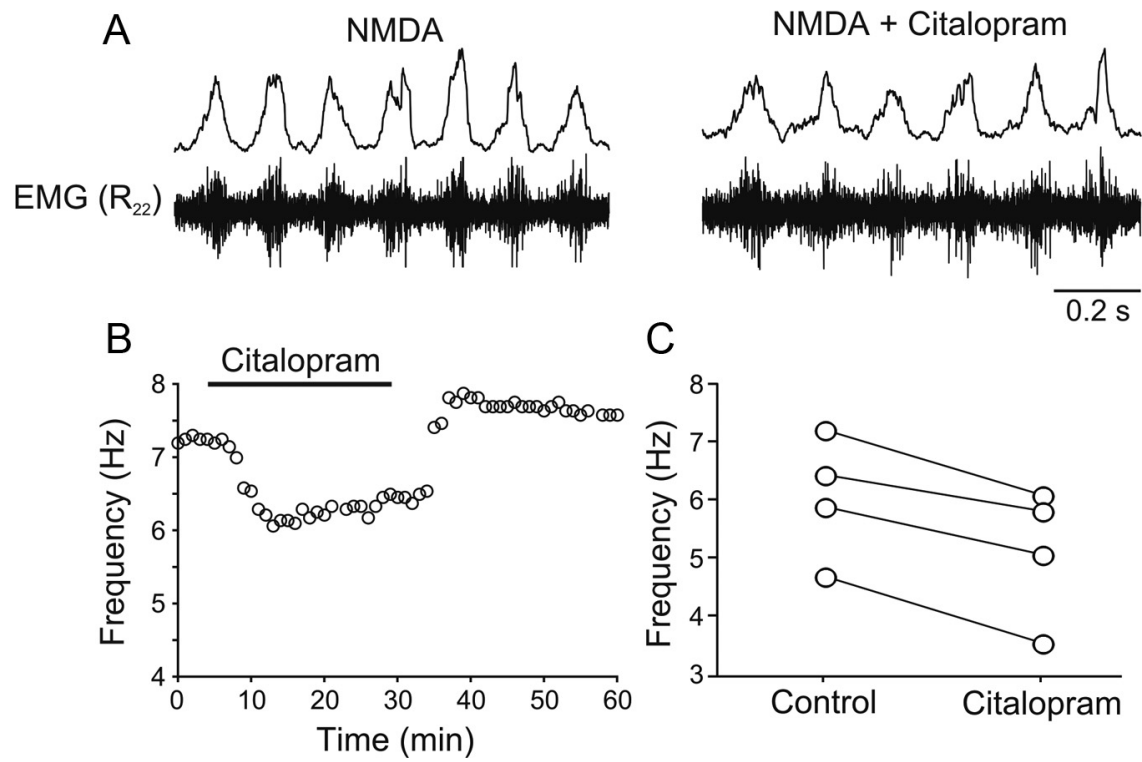

C

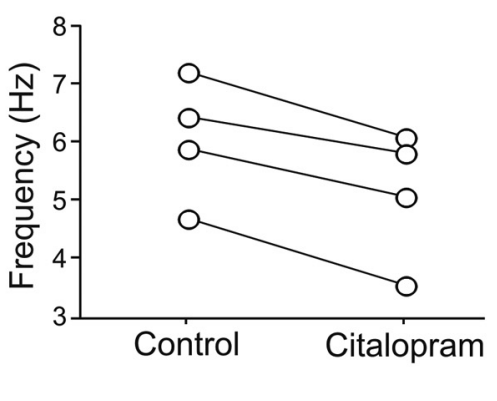

Figure 2. Endogenous release of 5-HT during locomotion. $A$, The frequency of the locomotor rhythm decreases by application of the selective serotonin reuptake inhibitor citalopram $(1-5 \mu \mathrm{M})$. $\boldsymbol{B}$, Time course of the decrease in the locomotor frequency (fictive locomotion induced by $40 \mu \mathrm{M}$ NMDA) by citalopram $(5 \mu \mathrm{M})$. C, Quantification of the decrease of the locomotor frequency (fictive locomotion induced by $40 \mu \mathrm{MNDA})$ in citalopram $(1-5 \mu \mathrm{M})(p<0.05 ; n=4)$.

dish. The dye-soaked pins were moved in the muscle to severe the motor axons and allow uptake of the dye. On the next day, the animals were dissected as described above for the patch-clamp recordings. Secondary motoneurons would be distinguished from primary mo- 
toneurons by their smaller soma size and more ventral soma position (Gabriel et al., 2008).

Electrophysiology. EMG recordings were performed from the myotomal muscles at the tail which were left intact during the preparation (Fig. 1A). Extracellular suction electrodes were pulled from borosilicate glass $[1 \mathrm{~mm}$ outer diameter (O.D.), $0.87 \mathrm{~mm}$ inner diameter (I.D.), Harvard Apparatus] on a Flaming/Brown microelectrode puller (P-87, Sutter Instruments), broken down to the desired tip diameter (15-25 $\mu \mathrm{M}$ ), and fire-polished. Extracellular signals were amplified (gain 10,000) with a differential AC amplifier (AM Systems) and filtered with low and high cutoff frequencies of $300 \mathrm{~Hz}$ and $1 \mathrm{kHz}$, respectively. Whole-cell patch-clamp recordings were performed from spinal neurons rostral to the EMG recording. Electrodes were pulled from borosilicate glass (1.5 $\mathrm{mm}$ O.D., $0.87 \mathrm{~mm}$ I.D.) and filled with intracellular solution containing in mM: $120 \mathrm{~K}$-gluconate, $5 \mathrm{KCl}, 10 \mathrm{HEPES}, 0.0001 \mathrm{CaCl}_{2}, 4 \mathrm{Mg}$-ATP, 0.3 $\mathrm{Na}$-GTP, $10 \mathrm{Na}_{2}$-phosphocreatine, with $\mathrm{pH}$ adjusted to 7.4 with $\mathrm{KOH}$, and osmolarity of $275 \mathrm{mOsm}$, yielding resistances of 5-8 M $\Omega$. Cells were visualized with a Zeiss Axioskop FS Plus upright microscope fitted with infrared differential interference contrast optics and a charge-coupled device camera with frame grabber (Hamamatsu). Intracellular signals were amplified with a MultiClamp 700B intracellular amplifier (Molecular Devices) and low-pass filtered at $10 \mathrm{kHz}$. In current-clamp recordings no bias current was injected.

Data acquisition and analysis. Data were digitized at $10 \mathrm{kHz}$ (extracellular recordings) or $40 \mathrm{kHz}$ (patch-clamp recordings) with a Digidata A/D converter (Molecular Devices) and acquired on a personal computer using pClamp software (version 9, Molecular Devices). Data analysis was performed in Spike2 (version 5, Cambridge Electronic Design). For the correlation analysis, extracellular traces were rectified, smoothed (time constant $0.01 \mathrm{~s}$ ) and down-sampled to $1 \mathrm{kHz}$ [smoothed rectified EMG (SR-EMG)]. The locomotor period was determined from the timeof-peak of the autocorrelation [for details, see the study by Gabriel et al. (2008)]. The burst proportion (duty-cycle) was calculated as the fraction of the cycle period occupied by a locomotor burst.

Unitary EPSCs and IPSCs were detected with MiniAnalysis software (version 6, Synaptosoft). At a holding potential of $0 \mathrm{mV}$, large outward currents could be recorded that corresponded to IPSCs (see Figs. 3C, 5B). At $-65 \mathrm{mV}$ we could record inward currents that corresponded both to fast EPSCs (in-phase with the ipsilateral EMG activity) and slower reversed IPSCs (out-of-phase with the ipsilateral EMG activity), which could be separated using a threshold for the decay time-constant of $0.8 \mathrm{~ms}$.

For the slope of the depolarization and hyperpolarization of the membrane potential of spinal neurons in strychnine, a linear regression was calculated for the data stretch spanning $30-70 \%$ of the depolarizing and hyperpolarizing waveform. An analysis of the residuals showed that time course of both the depolarization and the hyperpolarization was more or less linear at this point. The input resistance of spinal neurons was calculated as the slope of the linear region of the $V-I$ curve (membrane potentials between -90 and $-60 \mathrm{mV}$ ), which was obtained by injection of current pulses. All values are given as mean \pm SEM. The significance of differences of means between experimental groups and conditions was analyzed using Student's two-tailed $t$ test. Means were regarded as statistically significant at $p$ values of $<0.05$.

\section{Results}

Endogenous 5-HT decreases the frequency of

\section{fictive locomotion}

To examine the effect of 5-HT on the locomotor activity in juvenile and adult zebrafish, fictive locomotion was elicited in a brainstem/spinal cord preparation by superfusion of NMDA $(30-50 \mu \mathrm{M})$ (Fig. 1 B) (Gabriel et al., 2008). Application of 5-HT (1-20 $\mu \mathrm{M}$ ) reversibly decreased the locomotor frequency (Fig. $1 C, D)$. In $30 \mu \mathrm{M}$ NMDA, the frequency decreased from $4.9 \pm 0.4$ $\mathrm{Hz}$ to $3.8 \pm 0.4 \mathrm{~Hz}(5 \mu \mathrm{M} 5-\mathrm{HT}, p<0.001, n=8)$, while in $50 \mu \mathrm{M}$ NMDA it decreased from $5.6 \pm 0.3 \mathrm{~Hz}$ to $4.9 \pm 0.3 \mathrm{~Hz}(5 \mu \mathrm{M}$ 5-HT, $p<0.001, n=14$ ). The effect was concentrationdependent with a more pronounced decrease of the locomotor frequency at higher 5-HT concentrations (Fig. 1E). The decrease
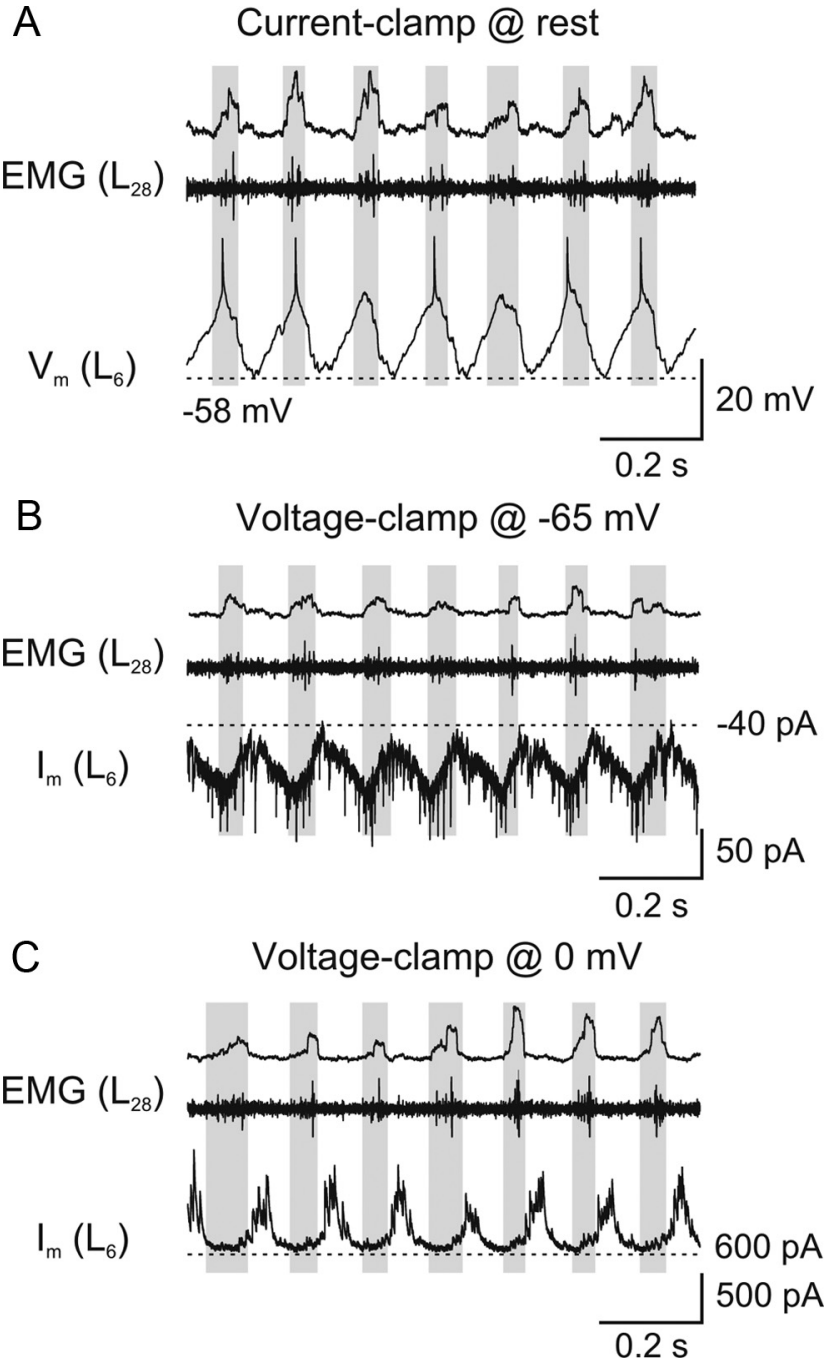

Figure 3. Phasic excitatory and inhibitory synaptic inputs during locomotion. $\boldsymbol{A}$, Intracellular recording from a spinal neuron which displays membrane potential oscillations during fictive locomotion induced by NMDA $(40 \mu \mathrm{m})$. This neuron depolarizes in phase with the ipsilateral, more caudal EMG recording (gray boxes). $\boldsymbol{B}$, When the neuron is clamped at the reversal potential of inhibitory currents $(-65 \mathrm{mV})$, EPSCs are visible and occur in phase with the ipsilateral EMG activity. $C$, When the neuron is clamped at the reversal potential of excitatory currents $(0 \mathrm{mV})$, IPSCs are visible and occur in antiphase with the EMG activity.

in the locomotor frequency by 5-HT was not associated with a change in the burst proportion (duty cycle), which was $0.24 \pm$ 0.03 in control and $0.26 \pm 0.03$ in 5 -HT $(p>0.05, n=6)$.

To test whether 5-HT is endogenously released and is intrinsically modulating the locomotor activity, the effect of the reuptake inhibitor citalopram was tested. Application of citalopram $(1-5 \mu \mathrm{M})$ decreased the locomotor frequency from $6.1 \pm 0.5 \mathrm{~Hz}$ to $5.2 \pm 0.6 \mathrm{~Hz}(p<0.05, n=4)$ (Fig. $2 A-C$ ), thus mimicking the effect of exogenous application of 5-HT. These results show that in the isolated zebrafish brainstem/ spinal cord 5-HT is endogenously released during locomotion and intrinsically modulates the ongoing locomotor activity.

\section{Spinal neurons receive phasic synaptic drive}

To examine possible synaptic mechanisms underlying the modulation of the locomotor frequency, we first sought to separate the excitatory and inhibitory inputs received by network neurons during locomotor activity. Spinal neurons show membrane potential oscillations during NMDA-induced fictive locomotion 

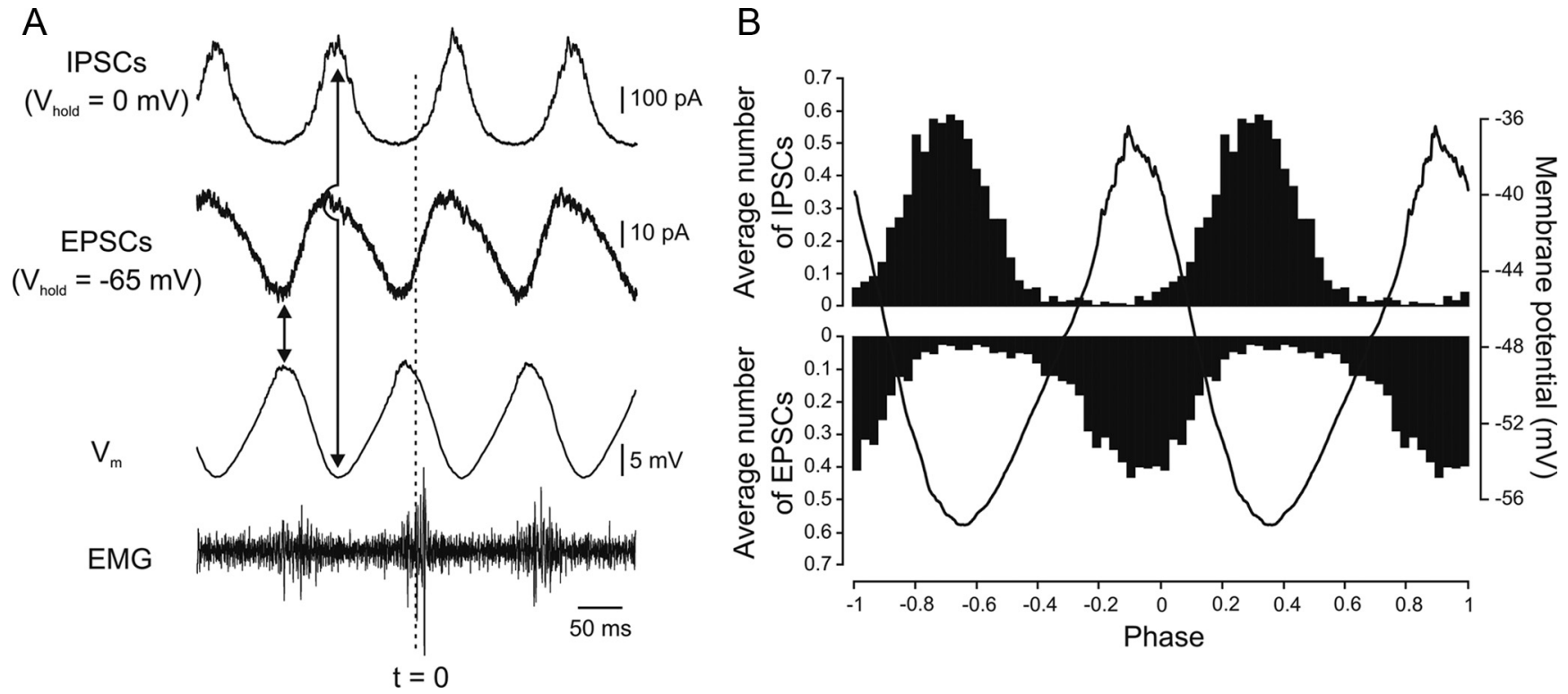

Figure 4. Alternating excitatory and inhibitory currents underlying the phasic oscillations during locomotion. $A$, Waveform average ( 50 swimming cycles each) of an intracellular recording from a spinal neuron during NMDA-induced fictive locomotion in current-clamp $\left(V_{m^{\prime}}\right.$, 0 bias current was injected) and voltage-clamp $\left(V_{\text {hold }}=0 \mathrm{mV}\right.$ and $\left.-65 \mathrm{mV}\right)$. The peak of the integrated ipsilateral EMG recording is taken as a reference $(t=0)$. $\boldsymbol{B}$, Histogram showing the distribution of the average number of unitary EPSCs and IPSCs received by the neuron during different phases of the locomotor cycle. The values correspond to the average number of PSCs per bin (binwidth 1/36th of a cycle corresponding to 10 degrees of phase) for 225 cycles for the IPSCs and 315 cycles for the EPSCS. The peak of the integrated ipsilateral EMG recording is used as a reference and the membrane potential oscillation is shown for comparison.

(Fig. 3A). All analyzed neurons were depolarized in phase with the ipsilateral EMG activity $(n=8)$. To separate the synaptic currents underlying the membrane potential modulation, neurons were voltage-clamped close to the reversal potential of excitation $(0 \mathrm{mV})$ or inhibition $(-65 \mathrm{mV})$ (Fig. $3 B, C)$. The neurons with the membrane potential clamped at $-65 \mathrm{mV}$ (close to the calculated reversal potential of inhibitory currents) displayed inward currents that occurred in phase with the ipsilateral EMG recording, i.e., in the depolarized phase in current-clamp (Fig. 3B). Similarly, inhibitory currents were isolated by clamping the membrane potential at 0 $\mathrm{mV}$ (the putative reversal potential of excitatory currents) and consisted of phasic outward currents occurring in anti-phase with the ipsilateral EMG burst, i.e., in the hyperpolarized phase in current-clamp (mid-cycle inhibition) (Fig. 3C).

The strict alternation of the phasic inward and outward currents was further corroborated by using a waveform average of the current traces recorded at $-65 \mathrm{mV}$ (EPSCs) and $0 \mathrm{mV}$ (IPSCs) together with the membrane potential oscillations. Because of the rostrocaudal delay of motor activity, the peak of the membrane potential $\left(V_{\mathrm{m}}\right)$ oscillations occurred before the peak of the SR-EMG, which was taken as reference point (Fig. $4 A$ ). The peak of the inward current coincided with the peak of the membrane potential oscillations, while that of the outward current coincided with the trough of the membrane potential oscillations.

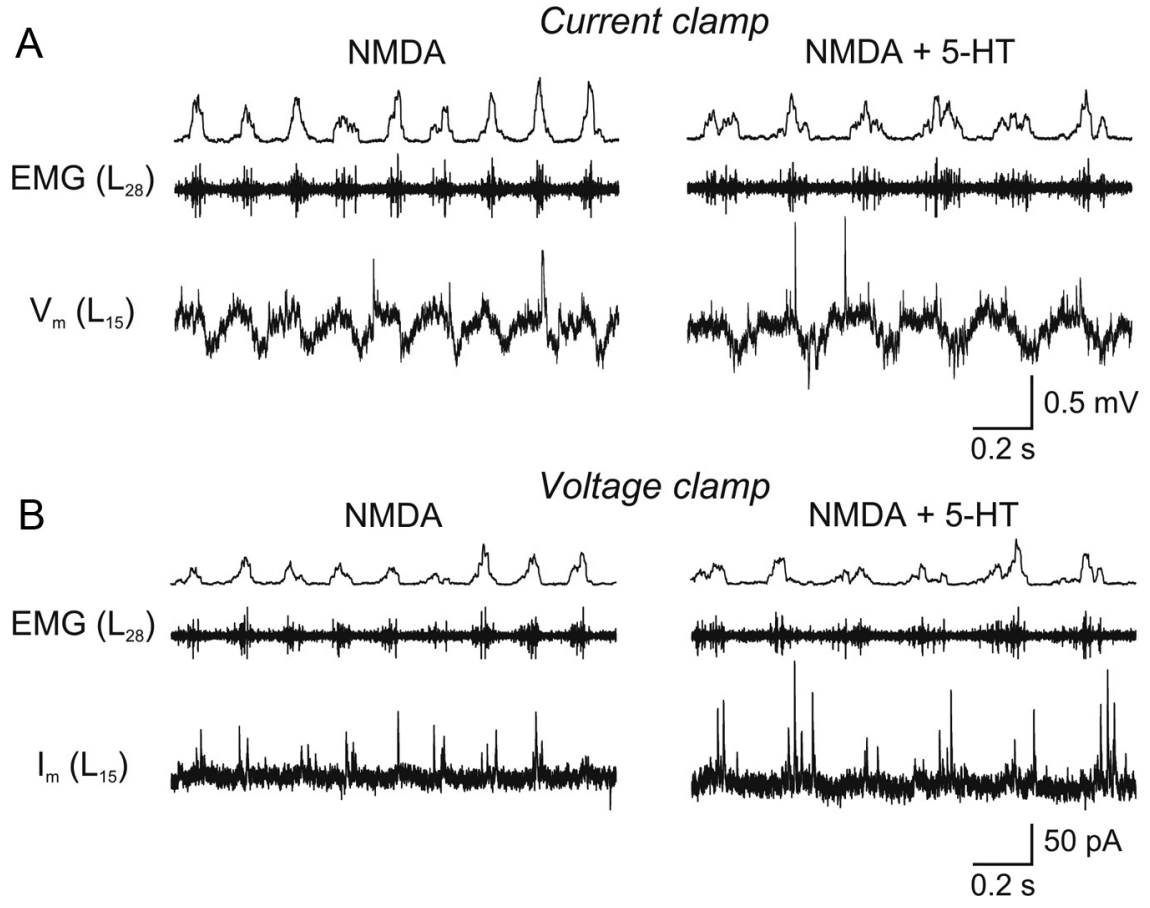

Figure 5. 5-HT increases the amplitude of mid-cycle inhibition during locomotion. $A$, Intracellular recording of a spinal neuron during fictive locomotion induced by NMDA $(40 \mu \mathrm{m})$ that shows membrane potential oscillations. Please note the occurrence of IPSPs during the hyperpolarized phase of the oscillations. In 5-HT (20 $\mu \mathrm{m})$, the locomotor frequency decreases and the IPSPs increase in amplitude and number. $\boldsymbol{B}$, Rhythmic IPSCs recorded in the same spinal neuron voltage-clamped at a holding potential of $0 \mathrm{mV}$ that increase in amplitude and number in $5-\mathrm{HT}(20 \mu \mathrm{m})$.

This shows that the on-phase excitation and mid-cycle inhibition are mediated by phasic and alternating excitatory and inhibitory synaptic currents, respectively. Furthermore, the analysis of individual PSCs showed that the highest EPSC frequency occurred at the peak of the membrane potential depolarization, while the highest IPSC frequency occurred at the 
A

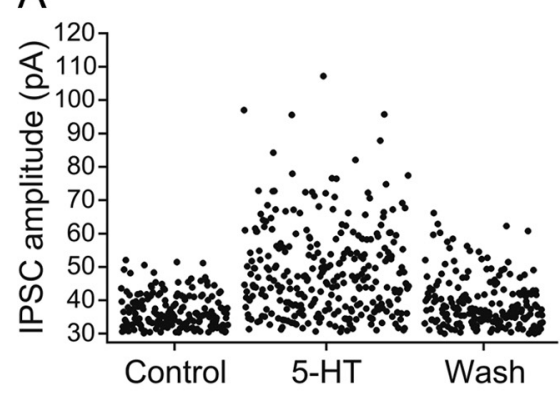

$\mathrm{C}$

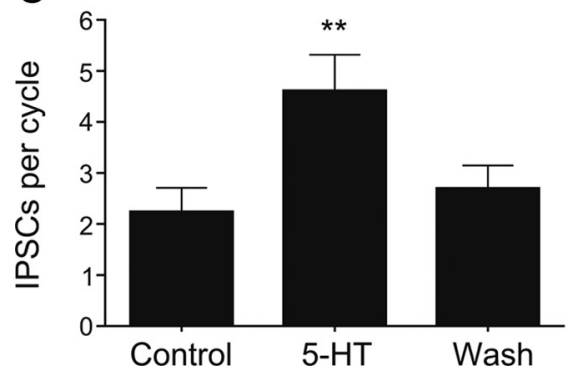

$\mathrm{B}$
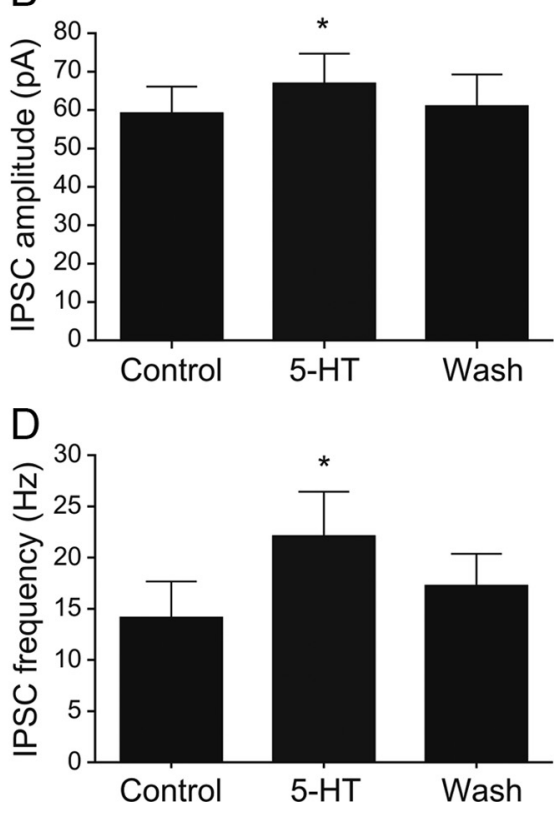

Figure 6. 5-HT increases the amplitude and frequency of IPSCs underlying mid-cycle inhibition. $A$, Amplitude of IPSCs recorded in a spinal cord neuron during locomotion in control (40 $\mu \mathrm{m} \mathrm{NMDA,} n=190$ events), 5-HT (10 $\mu \mathrm{m}$ in $40 \mu \mathrm{m} \mathrm{NMDA,} n=293$ events), and during washout ( $40 \mu \mathrm{m} \mathrm{NMDA}, n=210$ events). $\boldsymbol{B}$, The mean amplitude of the IPSC increases from $36.3 \pm 0.4 \mathrm{pA}$ in control to $48.2 \pm 0.8 \mathrm{pA}$ in 5-HT ( $p<0.0001$; washout: $38.9 \pm 0.5 \mathrm{pA}) . \mathbf{C}, \boldsymbol{D}$, Quantification of the change in the IPSC number per cycle $(p<0.01 ; n=8)$ and frequency $(p<0.05 ; n=8)$ in control, $5-\mathrm{HT}(10-20 \mu \mathrm{M})$, and wash.

most hyperpolarized membrane potential (Fig. $4 B$ ). In this cell there were on average 6.5 EPSCs and 7.0 IPSCs occurring during one locomotor cycle. These results clearly show that network neurons receive alternating phasic excitation and inhibition during locomotion.

\section{5-HT increases the phasic inhibition}

The discrimination between excitatory and inhibitory synaptic inputs during locomotion allowed us to examine the effect of 5-HT on each type of synaptic inputs separately. Under current-clamp conditions, the 5-HT-mediated decrease of the locomotor frequency was accompanied by an increase in amplitude and number of mid-cycle IPSPs (Fig. 5A). When the same neurons were voltage-clamped at $0 \mathrm{mV}, 5$-HT induced an increase in the amplitude of mid-cycle IPSCs (Fig. 5B). The amplitude and number of IPSCs were quantified over 150 swimming cycles in control and in 5-HT (Fig. 6A). On average, the IPSC amplitude increased from $59.2 \pm 6.9 \mathrm{pA}$ to $66.9 \pm 7.8 \mathrm{pA}(p<0.05, n=8$; washout: $61.0 \pm 8.3 \mathrm{pA})$ (Fig. $6 B)$. The number of IPSCs per cycle increased from $2.24 \pm$ 0.47 to $4.61 \pm 0.71(p<0.01, n=8$; washout: $2.70 \pm 0.45)$ (Fig. 6C). The IPSC frequency increased from $14.1 \pm 3.5 \mathrm{~Hz}$ to $22.1 \pm 4.3 \mathrm{~Hz}(p<0.05, n=8$; washout: $17.3 \pm 3.1 \mathrm{~Hz})$ (Fig. $6 D)$.

The effect of 5-HT on the EPSCs recorded in neurons voltageclamped at $-65 \mathrm{mV}$ was also analyzed (Fig. $7 A$ ). The mean EPSC amplitude and frequency did not change significantly in 5-HT (Fig. 7 B, C) (amplitude: control $19.2 \pm 2.0$ pA; 5-HT $18.4 \pm 1.3$ pA; washout $19.9 \pm 0.8 \mathrm{pA} ; p>0.05, n=6$; frequency: control $29.3 \pm 6.6 \mathrm{~Hz}, 5-\mathrm{HT} 33.0 \pm 9.0 \mathrm{~Hz}$, washout $35.9 \pm 10.9 \mathrm{~Hz} ; p>$ $0.05, n=6)$.

In addition to modulating synaptic transmission, 5-HT could also affect intrinsic cellular properties to decrease the locomotor frequency. We analyzed this in a defined population of spinal neurons, the dorsally located secondary motoneurons, which could be labeled and identified through backfills from the myotomal muscles (Gabriel et al., 2008). We measured the effect of 5-HT (10 $\mu \mathrm{M})$ on membrane potential and input resistance when action potentials were blocked by TTX $(0.5$ $\mu \mathrm{M})$ (Fig. 7D). 5-HT induced a small hyperpolarization of the resting membrane potential from $-65.5 \pm 0.8 \mathrm{mV}$ to $-66.7 \pm 0.6 \mathrm{mV}(p<0.05, n=6$; washout $-66.4 \pm 0.5 \mathrm{mV}$ ) without any detectable change in the input resistance that was $110.4 \pm 18.5 \mathrm{M} \Omega$ in control and $122.1 \pm 25.1 \mathrm{M} \Omega$ in 5 -HT $(p>$ $0.05, n=6$; washout: $116.7 \pm 22.4 \mathrm{M} \Omega$ ). Furthermore, 5-HT did not affect the $V-I$ relationship in these neurons (Fig. 7E). Application of NMDA in the presence of TTX did not induce any membrane oscillations in these secondary motoneurons neither in control nor in 5-HT.

These results show that 5-HT decreases the locomotor frequency by primarily increasing the amplitude and frequency of IPSCs underlying the mid-cycle inhibition, while the EPSCs mediating the phasic excitation as well as the basic cellular properties do not seem to be affected.

5-HT slows down the rhythmic activity induced in strychnine and changes the shape of the membrane potential oscillations To determine whether the modulation of the locomotor frequency by 5 -HT is solely mediated by an action on mid-cycle inhibition, the effect of 5-HT was tested after blockade of glycinergic synaptic transmission by strychnine $(0.5 \mu \mathrm{M})$. Strychnine switches the locomotor pattern from left-right alternation and rostrocaudal delay to a slow and synchronous pattern in different segments (Fig. 8A) (Gabriel et al., 2008). Application of 5-HT in the presence of strychnine decreased the frequency from $1.04 \pm$ $0.08 \mathrm{~Hz}$ to $0.77 \pm 0.05 \mathrm{~Hz}(p<0.001, n=12$; washout $1.00 \pm$ $0.08 \mathrm{~Hz}$ ) (Fig. $8 B, C)$.

To examine whether the decrease of the frequency of the rhythmic activity induced in strychnine is associated with a modulation of phasic synaptic inputs, we performed intracellular recordings from spinal neurons (Fig. 9A). The waveform average shows that the rising phase of the excitatory drive was slowed down with respect to the falling phase (Fig. 9B). This was quantified by calculating the ratio of the slope of the rising and the falling phase, which decreased from $1.14 \pm 0.13$ in control to $0.79 \pm 0.09$ in 5 -HT $(p<0.05, n=12$; washout $1.15 \pm 0.11)$ (Fig. 9C). The oscillation amplitude was not significantly changed in 5 -HT (control $3.7 \pm 1.1 \mathrm{mV} ; 5-\mathrm{HT} 4.3 \pm 1.2 \mathrm{mV}$; washout $3.6 \pm 0.9 \mathrm{mV} ; p>0.05, n=12)$. These results show that 5 -HT slows down the burst frequency the rhythmic activity even in the absence of glycinergic synaptic transmission by slowing the onset of the excitatory drive received by network neurons.

\section{Discussion}

\section{5-HT modulation in adult zebrafish spinal cord}

In the present study, we show that 5-HT is endogenously released within the spinal locomotor network of the adult/juvenile zebrafish and decreases the frequency of fictive swimming induced 
by NMDA. The 5-HT modulation of the frequency seems to be mediated by an enhancement of mid-cycle inhibitory synaptic transmission combined with a slowing down of the onset of the excitatory phase. Our results are in contrast to the reported effect of 5-HT in larval zebrafish (Brustein et al., 2003). At this early stage of development, 5-HT has no effect of the frequency of spontaneous locomotor activity and it only decreases the quiescent period between successive swimming episodes, resulting in an increase of swimming activity. It should be noted that the swimming frequency in larva is at least three times fasted than in the adult and that locomotor activity was induced pharmacologically in our study. 5-HT thus seems to modulate differently the activity of the spinal locomotor network in the larval and adult zebrafish.

Another important finding of this study is that the on-phase excitation and mid-cycle inhibition responsible for the locomotor pattern are mediated by phasic and alternating excitatory and inhibitory current, respectively. In the turtle spinal cord, it has been shown that the rhythmic activity underlying scratching induced by sensory stimulation does not involve phasic synaptic drives. In this system excitatory and inhibitory synaptic currents seem to occur simultaneously, and the resulting alternating depolarization and inhibition is caused by changes in the relative strength of the tonic currents (Berg et al., 2007). The occurrence of inhibitory synaptic potentials was previously demonstrated in the lamprey and mammalian spinal cord during NMDA-induced locomotion (Russell and Wallén, 1983) [see references in the study by Endo and Kiehn (2008)]. Our study confirms the fact that inhibitory synaptic currents are occurring phasically during locomotion and show that the excitatory currents are also phasic and alternating with the inhibition. It should be noted that scratching activity was induced by sensory stimulation while locomotion in our study and previous reports was induced pharmacologically.

\section{Endogenous release of 5-HT in zebrafish}

We investigated the possibility that $5-\mathrm{HT}$ is released endogenously during locomotion in zebrafish to modulate the ongoing locomotor activity. To achieve this, we applied the reuptake inhibitor citalopram, which increases the concentration of endogenous 5-HT in the synaptic cleft by inhibiting the transporter that is responsible for the rapid removal (Fuller and Wong, 1990) and thus enhances serotonergic neurotransmission. The fact that citalopram mimics the effects of 5-HT strongly suggests that there is endogenous release of 5-HT during NMDAinduced fictive locomotion in the isolated hindbrain/spinal cord preparation of juvenile and adult zebrafish. In larval zebrafish, the major serotonergic innervation of the spinal cord originates from the raphe region, which stretches from the
NMDA
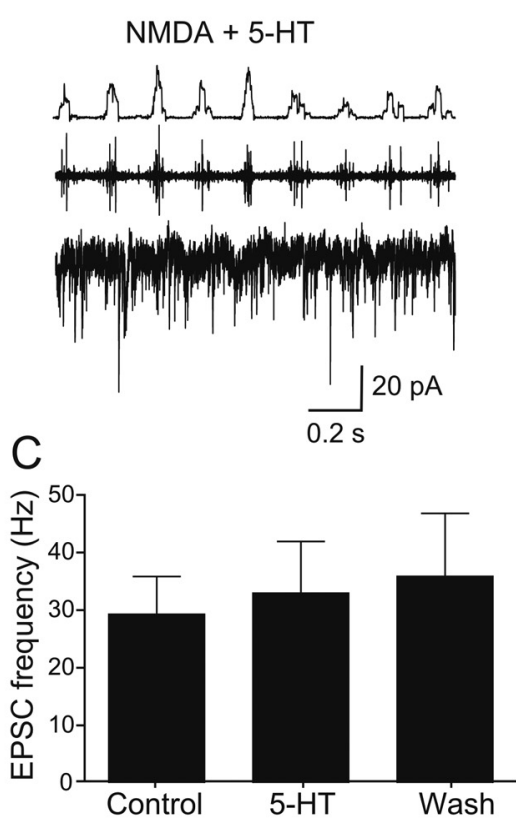

$E$

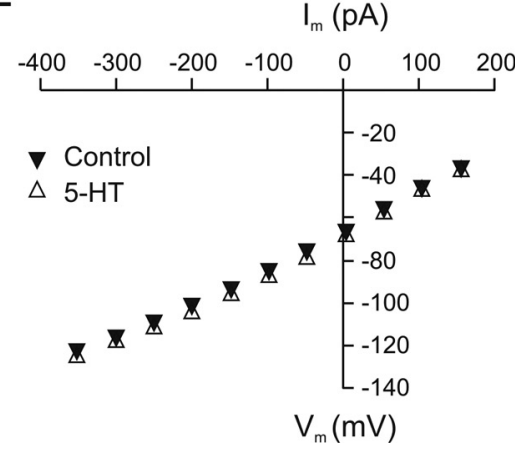

Figure 7. 5-HT does not affect the EPSCs underlying the phasic excitation or basic cellular properties. $\boldsymbol{A}$, Rhythmic EPSCS corded in a spinal neuron in voltage-clamp at a holding potential of $-65 \mathrm{mV}$ in control (40 $\mu \mathrm{m} \mathrm{NMDA)} \mathrm{and} \mathrm{in} \mathrm{5-HT} \mathrm{(10} \mu \mathrm{m})$. $\boldsymbol{B}$ motoneurons in the presence of TTX $(0.5 \mu \mathrm{m})$. Application of 5 -HT (10 $\mu \mathrm{m})$ did not change the input resistance. $\boldsymbol{E}$, The shape of the $\mathrm{V}-\mathrm{I}$ curve measured in dorsal secondary motoneurons was unaffected by 5 -HT.

caudal midbrain to the anterior medulla oblongata, and there are also intraspinal neurons positive for 5-HT (McLean and Fetcho, 2004; Brustein and Drapeau, 2005). This innervation pattern persists into adulthood (Van Raamsdonk et al., 1996; Kaslin and Panula, 2001), suggesting that 5-HT responsible for the modulation of the locomotor activity may arise from intraspinal neurons as well as supraspinal projections from the raphe.

\section{Influence of 5-HT on the locomotor network in} other preparations

In all major vertebrate preparations, 5 -HT has been found to modulate the spinal locomotor network (for review, see Sillar et al., 1998; Schmidt and Jordan, 2000). In Xenopus tadpoles, 5-HT has little effect on the swimming frequency (Sillar et al., 1992) but increases the intensity and duration of motor bursts mainly by depressing the synaptically driven mid-cycle inhibition of network neurons (McDearmid et al., 1997; McLean et al., 2000). In the lamprey spinal cord, 5-HT leads to a decrease in locomotor frequency and an increase in burst intensity and burst duration (Grillner and Wallén, 1980; Cohen and Harris-Warrick, 1984) through a depression of the slow afterhyperpolarization (sAHP) 
A

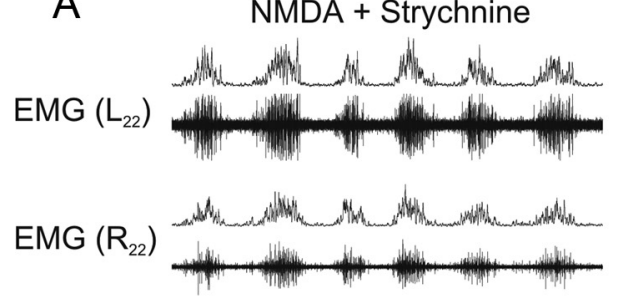

B

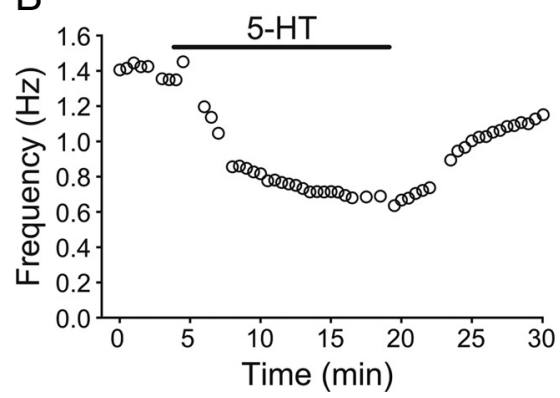

NMDA + Strychnine + 5-HT

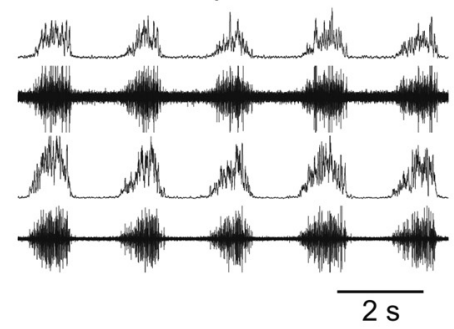

C

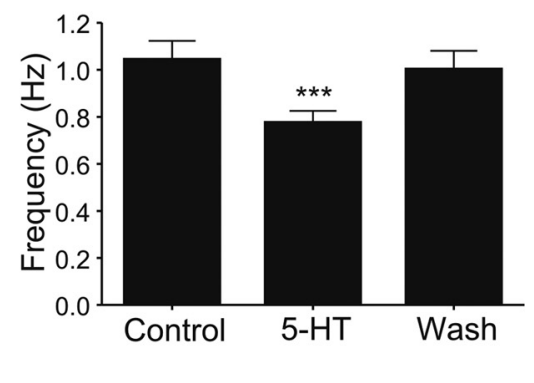

Figure 8. 5 -HT-induced decrease of the frequency of the rhythm in the absence of inhibition. $\boldsymbol{A}$, When glycinergic inhibition is blocked with strychnine $(0.5 \mu \mathrm{m}$ in $30-50 \mu \mathrm{M} \mathrm{NMDA})$, the alternating motor pattern changes to a slow, synchronous rhythm. Application of $5-\mathrm{HT}(5-10 \mu \mathrm{M})$ decreases the frequency of this rhythm. $\boldsymbol{B}$, Time course of the decrease in the rhythm frequency induced by $5-\mathrm{HT}(5 \mu \mathrm{m})$. C, Quantification of the decrease in the frequency by $5-\mathrm{HT}(5 \mu \mathrm{m} ; p<$ $0.001 ; n=12$ ).

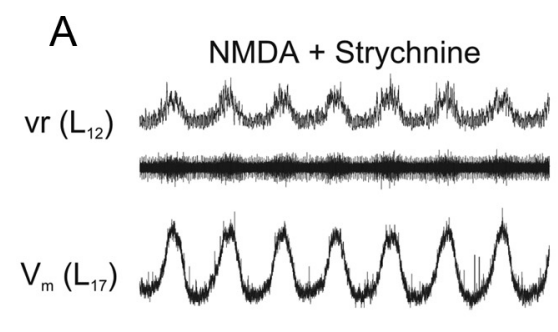

B

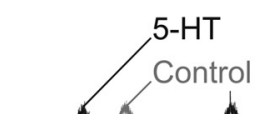

$\mathrm{V}_{\mathrm{m}}$
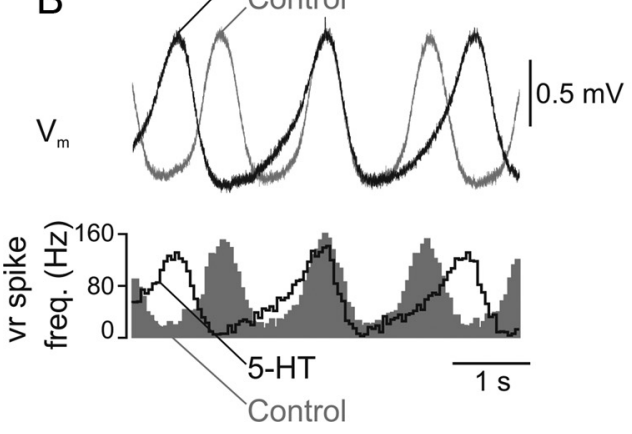
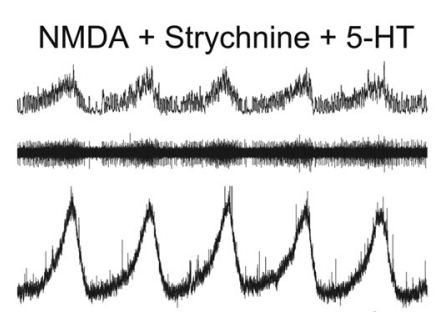

$1 \mathrm{mV}$

C

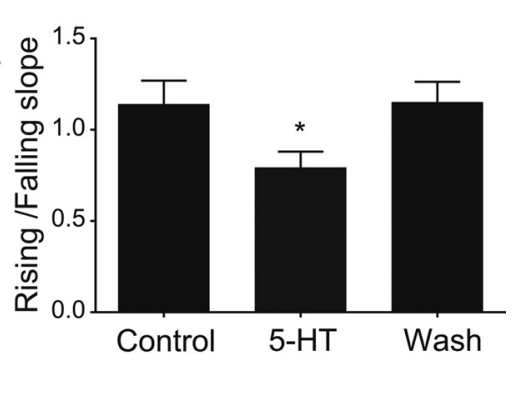

Figure 9. 5-HT delays the onset of the excitatory synaptic drive. A, When glycinergic inhibition is blocked by strychnine $(0.5 \mu \mathrm{M})$, NMDA $(50 \mu \mathrm{m})$ induces slow membrane potential oscillations in spinal neurons that occur in phase with the ipsilateral ventral root burst. Application of 5-HT $(10 \mu \mathrm{m})$ decreases the burst frequency and slows down the depolarizing phase of the excitatory drive. $\boldsymbol{B}$, Waveform average of 25 cycles in control and in 5-HT from the same recording as in $\boldsymbol{A}$. The bottom trace shows the average spike frequency of the ipsilateral ventral root in control (gray area) and in 5-HT (black outline). $\boldsymbol{C}$, The slope of the linear fit to the depolarizing and the repolarizing phase ( $30-70 \%$ amplitude) was calculated. The ratio of these slopes decreases in 5-HT $(5-10 \mu \mathrm{m})$, indicating that the depolarization is slowed down with respect to the repolarization phase $(p<0.05, n=12)$.

(Wallén et al., 1989b) and modulation of synaptic transmission (Biró et al., 2006). In the in vitro spinal cord preparation of the neonatal rat and mouse, 5-HT alone induces fictive locomotion (Cazalets et al., 1992; Kiehn and Kjaerulff, 1996; Schmidt and Jordan, 2000) and, depending on the receptors activated, it decreases or increases the frequency of the NMDAinduced locomotor rhythm and increases its burst amplitude and duration (Beato and Nistri, 1998; Bracci et al., 1998; Madriaga et al., 2004). In addition, 5-HT has been shown to affect the firing properties of motoneurons and commissural interneurons (Kjaerulff and Kiehn, 2001; Zhong et al., 2006a,b).

The differential modulation of the locomotor activity at early and late developmental stages in zebrafish is analogous to that reported in Xenopus. In embryos, 5-HT only affects the burst duration without change in the frequency (Sillar et al., 1992), while in premetamorphic tadpoles it decreases the frequency (Rauscent et al., 2009). The mechanisms responsible for the change in the actions of 5-HT during development are not yet clear. These may include a change in the expression of 5-HT receptors and their location on soma/dendrites or on axon terminals. Another possibility could be the developmental changes in the expression of the type of ion channels modulated by 5 -HT that can affect the firing properties of neurons and synaptic transmission (Sun and Dale, 1998; Jiménez-González et al., 2003).

5-HT targets the mid-cycle inhibition to modulate the locomotor pattern

In this study, we show that 5-HT increases the mid-cycle inhibition spinal neurons receive during fictive locomotion induced by NMDA. Computer simulations have shown that an increase of the mid-cycle inhibition can effectively decrease the locomotor frequency by delaying activation of the contralateral side (Hellgren et al., 1992; Dale, 1995). In Xenopus tadpole and lamprey, 5-HT reduces the mid-cycle inhibition (McDearmid et al., 1997; Biró et al., 2006). In contrast, we show that in the adult zebrafish 5-HT potentiates inhibitory synaptic transmission during fictive locomotion and slows down the onset of the excitatory drive. These effects can result in a decrease in the locomotor frequency induced by endogenously released 5-HT. In larval zebrafish, the decrease of the quiescence period by 5 -HT was shown to be mediated by an effect on the chloride homeostasis and as a result a decrease in the inhibitory glycinergic synaptic transmission (Brustein et al., 2003). This effect is absent in the adult zebrafish because 5-HT potentiates rather than depresses inhibition, and its effect on the rhythm frequency is not abolished after blockade of glycinergic synaptic transmission. 


\section{Effect of 5-HT after blockage of glycinergic neurotransmission}

In adult zebrafish, the modulation of the locomotor activity by 5-HT did not solely involve an effect on inhibitory synaptic transmission, because a decrease in the locomotor frequency was also seen in the presence of the glycine receptor antagonist strychnine. In these experiments, the decrease in the frequency of the rhythmic activity induced in strychnine was associated with a slowing down of the onset of the excitatory drive received by motoneurons. This indicates that 5-HT acts on both the excitatory and inhibitory synaptic transmission to modulate the activity of the spinal network. The exact mechanisms by which 5-HT delays the excitatory drive have not been examined in zebrafish. However, 5-HT has been shown to modulate the NMDA response in lamprey (Zhang et al., 1996), the Xenopus tadpole (ScrymgeourWedderburn et al., 1997) and the rat spinal cord (MacLean et al., 1998). It is likely that in the zebrafish spinal cord 5-HT exerts a similar effect on NMDA receptors to slow down the depolarizing phase, but we cannot rule out the possibility that this is caused by a decrease in synaptic excitation. Although intrinsic oscillations have not yet been demonstrated in zebrafish, part of the membrane potential oscillations observed in strychnine may be generated by bistable membrane properties.

In conclusion, we have shown that 5-HT exerts a different role in more mature zebrafish than early in development in the larva. 5-HT is endogenously released during locomotor activity and decreases the burst frequency in parallel with an increase in the mid-cycle inhibition. It will be important in future experiments to determine the mechanisms responsible for the switch of the effect of 5-HT as the zebrafish matures to adulthood.

\section{References}

Beato M, Nistri A (1998) Serotonin-induced inhibition of locomotor rhythm of the rat isolated spinal cord is mediated by the 5-HT1 receptor class. Proc Biol Sci 265:2073-2080.

Berg RW, Alaburda A, Hounsgaard J (2007) Balanced inhibition and excitation drive spike activity in spinal half-centers. Science 315:390-393.

Biró Z, Hill RH, Grillner S (2006) 5-HT Modulation of identified segmental premotor interneurons in the lamprey spinal cord. J Neurophysiol 96:931-935.

Bracci E, Beato M, Nistri A (1998) Extracellular K+ induces locomotor-like patterns in the rat spinal cord in vitro: comparison with NMDA or 5-HT induced activity. J Neurophysiol 79:2643-2652.

Brustein E, Drapeau P (2005) Serotoninergic modulation of chloride homeostasis during maturation of the locomotor network in zebrafish. J Neurosci 25:10607-10616.

Brustein E, Chong M, Holmqvist B, Drapeau P (2003) Serotonin patterns locomotor network activity in the developing zebrafish by modulating quiescent periods. J Neurobiol 57:303-322.

Cazalets JR, Sqalli-Houssaini Y, Clarac F (1992) Activation of the central pattern generators for locomotion by serotonin and excitatory amino acids in neonatal rat. J Physiol 455:187-204.

Cohen AH, Harris-Warrick RM (1984) Strychnine eliminates alternating motor output during fictive locomotion in the lamprey. Brain Res 293:164-167.

Dale N (1995) Experimentally derived model for the locomotor pattern generator in the Xenopus embryo. J Physiol 489:489-510.

El Manira A, Zhang W, Svensson E, Bussières N (1997) 5-HT inhibits calcium current and synaptic transmission from sensory neurons in lamprey. J Neurosci 17:1786-1794.

Endo T, Kiehn O (2008) Asymmetric operation of the locomotor central pattern generator in the neonatal mouse spinal cord. J Neurophysiol 100:3043-3054.

Fetcho JR, Higashijima S, McLean DL (2008) Zebrafish and motor control over the last decade. Brain Res Rev 57:86-93.

Fuller RW, Wong DT (1990) Serotonin uptake and serotonin uptake inhibition. Ann N Y Acad Sci 600:68-78.
Gabriel JP, Mahmood R, Walter AM, Kyriakatos A, Hauptmann G, Calabrese RL, El Manira A (2008) Locomotor pattern in the adult zebrafish spinal cord in vitro. J Neurophysiol 99:37-48.

Grillner S, Wallén P (1980) Does the central pattern generation for locomotion in lamprey depend on glycine inhibition? Acta Physiol Scand 110:103-105.

Hellgren J, Grillner S, Lansner A (1992) Computer simulation of the segmental neural network generating locomotion in lamprey by using populations of network interneurons. Biol Cybern 68:1-13.

Jiménez-González C, McLaren GJ, Dale N (2003) Development of $\mathrm{Ca}^{2+}$. channel and BK-channel expression in embryos and larvae of Xenopus laevis. Eur J Neurosci 18:2175-2187.

Kaslin J, Panula P (2001) Comparative anatomy of the histaminergic and other aminergic systems in zebrafish (Danio rerio). J Comp Neurol 440:342-377.

Kiehn O, Kjaerulff O (1996) Spatiotemporal characteristics of 5-HT and dopamine-induced rhythmic hindlimb activity in the in vitro neonatal rat. J Neurophysiol 75:1472-1482.

Kjaerulff O, Kiehn O (2001) 5-HT modulation of multiple inward rectifiers in motoneurons in intact preparations of the neonatal rat spinal cord. J Neurophysiol 85:580-593.

Liu J, Jordan LM (2005) Stimulation of the parapyramidal region of the neonatal rat brain stem produces locomotor-like activity involving spinal 5-HT7 and 5-HT2A receptors. J Neurophysiol 94:1392-1404.

Liu J, Akay T, Hedlund PB, Pearson KG, Jordan LM (2009) Spinal 5-HT7 receptors are critical for alternating activity during locomotion: in vitro neonatal and in vivo adult studies using 5-HT7 receptor knockout mice. J Neurophysiol 102:337-348.

MacLean JN, Cowley KC, Schmidt BJ (1998) NMDA receptor-mediated oscillatory activity in the neonatal rat spinal cord is serotonin dependent. J Neurophysiol 79:2804-2808.

Madriaga MA, McPhee LC, Chersa T, Christie KJ, Whelan PJ (2004) Modulation of locomotor activity by multiple 5-HT and dopaminergic receptor subtypes in the neonatal mouse spinal cord. J Neurophysiol 92:1566-1576.

Masino MA, Fetcho JR (2005) Fictive swimming motor patterns in wild type and mutant larval zebrafish. J Neurophysiol 93:3177-3188.

McDearmid JR, Drapeau P (2006) Rhythmic motor activity evoked by NMDA in the spinal zebrafish larva. J Neurophysiol 95:401-417.

McDearmid JR, Scrymgeour-Wedderburn JF, Sillar KT (1997) Aminergic modulation of glycine release in a spinal network controlling swimming in Xenopus laevis. J Physiol 503:111-117.

McLean DL, Fetcho JR (2004) Ontogeny and innervation patterns of dopaminergic, noradrenergic, and serotonergic neurons in larval zebrafish. J Comp Neurol 480:38-56.

McLean DL, Merrywest SD, Sillar KT (2000) The development of neuromodulatory systems and the maturation of motor patterns in amphibian tadpoles. Brain Res Bull 53:595-603.

Rauscent A, Einum J, Le Ray D, Simmers J, Combes D (2009) Opposing aminergic modulation of distinct spinal locomotor circuits and their functional coupling during amphibian metamorphosis. J Neurosci 29:1163-1174.

Russell DF, Wallén P (1983) On the control of myotomal motoneurones during "fictive swimming" in the lamprey spinal cord in vitro. Acta Physiol Scand 117:161-170.

Schmidt BJ, Jordan LM (2000) The role of serotonin in reflex modulation and locomotor rhythm production in the mammalian spinal cord. Brain Res Bull 53:689-710.

Schwartz EJ, Gerachshenko T, Alford S (2005) 5-HT prolongs ventral root bursting via presynaptic inhibition of synaptic activity during fictive locomotion in lamprey. J Neurophysiol 93:980-988.

Scrymgeour-Wedderburn JF, Reith CA, Sillar KT (1997) Voltage oscillations in Xenopus spinal cord neurons: developmental onset and dependence on co-activation of NMDA and 5HT receptors. Eur J Neurosci 9:1473-1482.

Sillar KT, Wedderburn JF, Simmers AJ (1992) Modulation of swimming rhythmicity by 5-hydroxytryptamine during post-embryonic development in Xenopus laevis. Proc Biol Sci 250:107-114.

Sillar KT, Reith CA, McDearmid JR (1998) Development and aminergic neuromodulation of a spinal locomotor network controlling swimming in Xenopus larvae. Ann N Y Acad Sci 860:318-332.

Sun Q, Dale N (1998) Developmental changes in expression of ion currents 
accompany maturation of locomotor pattern in frog tadpoles. J Physiol 507:257-264.

Van Raamsdonk W, Bosch TJ, Smit-Onel MJ, Maslam S (1996) Organisation of the zebrafish spinal cord: distribution of motoneuron dendrites and 5-HT containing cells. Eur J Morphol 34:65-77.

Wallén P, Christenson J, Brodin L, Hill R, Lansner A, Grillner S (1989a) Mechanisms underlying the serotonergic modulation of the spinal circuitry for locomotion in lamprey. Prog Brain Res 80:321-327.

Wallén P, Buchanan JT, Grillner S, Hill RH, Christenson J, Hökfelt T (1989b) Effects of 5-hydroxytryptamine on the afterhyperpolarization, spike frequency regulation, and oscillatory membrane properties in lamprey spinal cord neurons. J Neurophysiol 61:759-768.
Westerfield M (2000) The zebrafish book. A guide for the laboratory use of zebrafish (Danio rerio), Ed 4. Eugene, OR: University of Oregon.

Zhang W, Pombal MA, el Manira A, Grillner S (1996) Rostrocaudal distribution of 5-HT innervation in the lamprey spinal cord and differential effects of 5-HT on fictive locomotion. J Comp Neurol 374:278-290.

Zhong G, Díaz-Ríos M, Harris-Warrick RM (2006a) Intrinsic and functional differences among commissural interneurons during fictive locomotion and serotonergic modulation in the neonatal mouse. J Neurosci 26:6509-6517.

Zhong G, Díaz-Ríos M, Harris-Warrick RM (2006b) Serotonin modulates the properties of ascending commissural interneurons in the neonatal mouse spinal cord. J Neurophysiol 95:1545-1555. 